Voix et Images

voixetimages

\title{
Roger Des Roches : le pont surréaliste entre le cul et l'écriture
}

\section{André Marquis}

Volume 13, numéro 2 (38), hiver 1988

Le propre du corps Roger Des Roches

URI : https://id.erudit.org/iderudit/200709ar

DOI : https://doi.org/10.7202/200709ar

Aller au sommaire du numéro

\section{Éditeur(s)}

Université du Québec à Montréal

\section{ISSN}

0318-9201 (imprimé)

1705-933X (numérique)

Découvrir la revue

\section{Citer cet article}

Marquis, A. (1988). Roger Des Roches : le pont surréaliste entre le cul et l'écriture. Voix et Images, 13(2), 270-279. https://doi.org/10.7202/200709ar d'utilisation que vous pouvez consulter en ligne.

https://apropos.erudit.org/fr/usagers/politique-dutilisation/ 


\title{
Roger Des Roches: le pont surréaliste entre le cul et l'écriture1
}

\section{par André Marquis, Université de Sherbrooke}

\author{
À R.G., J.B., S.C., J.M. et D.G., sans qui \\ ce texte n'aurait pas vu le jour - plus \\ sérieusement à la mémoire de Suzanne \\ Lamy, puisque l'ensemble de nos recherches \\ ont été effectuées dans le cadre d'un séminaire \\ de maitrise et de doctorat qu'elle donna à \\ l'Université de Sherbrooke à l'hiver 1986.
}

L'aventure des Herbes rouges débutait, bien modestement, en 1968 par la publication de petits numéros de revues consacrés à la poésie moderne; depuis lors, non seulement les Herbes rouges sont devenues une véritable maison d'édition, mais elles représentent l'un des lieux les plus dynamiques de l'avantgarde poétique québécoise. Depuis quelque temps, la reconnaissance a dépassé le cercle restreint des poètes puisque, en 1986, André Roy et Normand de Bellefeuille ont remporté deux prix littéraires d'importance. Qu'on soit d'accord ou non avec leur esthétique, les auteurs des Herbes rouges ont acquis, depuis quinze ans, une grande maîtrise de l'écriture poétique et se sont taillé une place enviable dans le champ littéraire québécois.

Les premiers noms qui nous viennent à l'esprit lorsqu'on pense à cette maison d'édition sont François Charron, André Roy, France Théoret et Normand de Bellefeuille. Et le poète que l'on oublie le plus souvent est Roger Des Roches, pourtant un des producteurs les plus assidus depuis les tout débuts. Curieux itinéraire que celui de Roger Des Roches! Considéré comme un des piliers des Herbes rouges, il est à l'écoute de courants aussi différents que la contreculture, le formalisme, la psychanalyse et le surréalisme. Malgré la publication de 18 titres depuis 1970, la critique québécoise ne semble pas disposée à le placer à son juste rang. À l'écoute de la littérature américaine, Des Roches conjugue la science-fiction à l'ironie grâce à une imagination déchaînée. À maintes reprises, il a perturbé le champ poétique par une production innovatrice, en rupture avec les codes établis. Mentionnons l'insertion de parenthèses, les jeux calligraphiques, les notes en bas de page, les bribes de langue parlée; voilà autant de

1 Ce texte est une version remaniée d'une communication présentée le 6 mars 1987 à l'Université du Québec à Montréal dans le cadre du minicolloque sur les Herbes rouges organisé par Cécile Cloutier de l'Université de Toronto. 
procédés qui ont été repris par de nombreux auteurs. Ainsi en va-t-il de Des Roches: ses livres marquent les producteurs, mais ne reçoivent presque jamais l'attention de la critique.

Des Roches a publié ses deux premiers recueils de poésie, Corps accessoires et l'Enfance d'yeux suivi de Interstice, aux Éditions du Jour en 1970 et 1972. L'année suivante, en 1973, il fait paraître les Problèmes du cinématographe dans la revue les Herbes rouges, qui présentait ainsi un de ses premiers numéros d'auteur. Ce livre a énormément plu à André Roy et à Normand de Bellefeuille, parce qu'il reposait sur une structure cohérente et qu'il incorporait en poésie un langage jusqu'alors réservé au domaine cinématographique. La même année, dans le numéro 15 de la revue, Des Roches tente une nouvelle expérience et offre au lecteur un récit (?) de science-fiction intitulé SPACE-OPERA (sur-exposition). L'année suivante, il publie aux éditions de l'Aurore, Reliefs de l'Arsenal, où il traite plus longuement de la confusion des genres. En 1975, paraît Autour de Françoise Sagan indélébile dans la collection «Lecture en vélocipède», dont s'occupaient les frères Hébert aux éditions de l'Aurore et qu'ils poursuivront, en 1978, sous la bannière des Herbes rouges. Des Roches participe régulièrement aux numéros d'auteur de la revue et produit, de 1975 à 1979, six textes d'une trentaine de pages chacun ${ }^{2}$. L'année 1979 marque une rupture dans la production de Des Roches. Il fait alors paraître deux recueils très différents: d'abord sa rétrospective, «Tous, corps accessoires...», qui regroupe ses premiers textes 3 ; puis, Pourvu que ça ait mon nom, un livre écrit en collaboration avec. Normand de Bellefeuille et dans lequel il se tourne vers un univers prosaïque plus personnel. Par la suite, Des Roches publie à un rythme un plus lent: l'Imagination laïque, en 1982 , dans la collection «Lecture en vélocipède», Poème, attention! suivi de Deuxième poème, dans le numéro 128 des Herbes rouges, en 1984, et son dernier livre, le Soleil tourne autour de la terre, en 1985. Entre ces deux dernières publications, l'auteur a conçu, avec trois autres personnes, un système de traitement de texte lequel, curieusement, intéressera davantage les critiques que ses livres de poésie.

Sans reprendre systématiquement chaque recueil, pointons les préoccupations dominantes de cet auteur. On peut déjà affirmer que les premières œuvres de Des Roches sont largement influencées par le surréalisme, tandis que les suivantes le sont beaucoup moins. Cependant, tout au long de son cheminement poétique; Des Roches demeure fidèle à deux principes d'écriture: le travail formel et l'exploitation de la sexualité comme isotopie dominante. Il écrira, dans l'Imagi-

2 Il s'agit de: la Publicité discrète, $\mathrm{n}^{\circ}$ 25, 1975, 28 p.; le Corps certain, $\mathrm{n}^{\circ} 30,1975,24$ p.; la Vle de couple, $\mathrm{n}^{\circ} \mathrm{s} 50-51,1977$, [n.p.]; la Promenade du spécialiste, $\mathrm{n}^{\circ} 54,1977,24$ p.; les Lèvres de $n^{\prime}$ importe qui, $\mathrm{n}^{\circ} 70,1978,31 \mathrm{p}$; l'Observatoire romanesque, $\mathrm{n}^{\circ} 77$, 1979,29 p.

3 A savoir: Corps accessoires, l'Enfance d'yeux suivi de Interstice, les Problèmes du cinématographe, SPACE-OPERA (surexposition) et Autour de Françoise Sagan indélébile. 
nation laïque, un vers qui résume bien cette quête (d'où le titre de notre article): le pont surréaliste entre le cul et l'écriture sauve la langue. Des Roches a aussi exploré la fiction narrative, en prenant ses distances face aux codes usuels du récit.

\section{Assurément sourire surréaliste}

L'influence surréaliste de Des Roches est reconnue dès son premier recueil, Corps accessoires. Jean Basile, dans une chronique du Devoir ${ }^{4}$, établit cette filiation, sans développer davantage. À quoi s'apparentent donc ces Corps accessoires? Tout d'abord, si l'épigraphe présente un vers de Tristan Tzara 5 , le recueil nous apparaît fortement imprégné du Paul-Marie Lapointe première manière. Les images surprenantes et parfois même abracadabrantes, la crudité de certaines descriptions (notons une affection particulière du narrateur pour les seins), tout cela enrobé d'une ironie provocatrice, ne sont pas sans rappeler le Vierge incendié. Par exemple: face contre terre aux pieds des pieds alpestres de grand-mères béatiques (p. 19), ou encore: débitant des vers d'après vierge [...] dans son crâne (p. 21). Rappelons qu'à l'endos de la rétrospective, apparaît un vers de Paul-Marie Lapointe. Ce dernier, ainsi que Roland Giguère, ont collaboré d'ailleurs aux premiers numéros de la revue les Herbes rouges.

Des Roches aime puiser ses images dans ce que la culture nord-américaine a de plus banal, de plus quotidien. Ainsi, les marques de sous-vêtements «WonderBra» et «Mr. Brief» deviennent prétexte à métaphores, à jeux de mots plus ou moins réussis. Il y a une recherche sonore un peu simpliste dans ce premier recueil, qui en limite la portée. Que ce soit un mot, électro-Lise (p. 43), ou des vers entiers qui frôlent la facilité: agoraphobique Attila lubrique dans la brique (p. 40). On perçoit chez Des Roches un attrait certain pour les recherches phonétiques à la Claude Gauvreau, et il s'en donnera à cœur joie dans Autour de Françoise Sagan indélébile. Un aspect intéressant de Corps accessoires réside sûrement dans la présence de vers ou de bribes de vers écrits en anglais. Cette tendance s'accentuera d'ailleurs dans les recueils subséquents pour aboutir à des suites complètes de quelques pages. Ce premier livre fourmille donc d'influences diverses. Les préoccupations formalistes du poète vont se cristalliser dès son deuxième titre, l'Enfance d'yeux.

Dans ce recueil, Des Roches exploite essentiellement deux filons du code poétique: d'une part, la dimension sonore (allitérations, assonances et paronomases), d'autre part, la déconstruction syntaxique. Les poèmes passent d'un pro-

4 Jean Basile, «Publication de trois 'jeunes poètes' [Jacques Boulerice, Louis Geoffroy, Roger Des Roches]», le Devoir, 29 septembre 1970, p. 12.

5 Car jamais parole n'a franchi le seuil des corps, l'Homme approximatif (1931). Notons la présence du mot «corps» qui revient aussi dans le titre du recueil de Des Roches. En 1975, il reprendra d'ailleurs ce terme pour un autre recueil de poésie, le Corps certain. Est-il besoin de rappeler le rôle important que jouera le mot «corps», tout au long des années soixante-dix, dans la production poétique québécoise? 
cédé à l'autre dans une visée plus expérimentale que cohérente. Parfois, l'auteur s'élance sur une piste jonchée de mots-valises et de néologismes; à d'autres occasions, il poursuit des recherches plus formelles en utilisant les signes de ponctuation ou la calligraphie. Le lecteur est frappé par l'aspect chamboulé du livre:

\author{
mes mille mains moins maintes mains d'housses \\ c'est ce corps craint qui crible \\ l'outre temporale de mon front feint
}

ta salive soude mon souffle

à toute l'étendue de ton clavesein (p. 87)

Interstice, son troisième recueil, est plutôt un travail d'expérimentation. Des Roches rédige de courts poèmes, cherchant à éliminer l'effet de répétition. L'écriture devient par conséquent beaucoup plus serrée et elliptique. Pour que l'attention du lecteur porte essentiellement sur le jeu de déconstruction syntaxique, Des Roches élimine tout ce qui pourrait «nuire» au décodage: jeux de mots faciles, signes de ponctuation, vers en anglais. Il sélectionne judicieusement ses mots et veille à ne pas produire d'effet ironique:

\author{
plonge \\ comme retrace l'iridescence \\ si tu te soucies moins du sol \\ plus ne sais que te rapprochant (p. 115)
}

Au premier abord, le lecteur est désorienté s'il ne comprend que les divers marqueurs du discours (embrayeurs, marqueurs logico-argumentatifs, etc.) portent moins sur le contenu des énoncés que sur leur énonciation. Ces marqueurs articulent en effet le dit avec le non-dit, le signe graphique avec l'espace blanc. D'où la nécessité de rompre avec nos habitudes de lecture et de chercher nousmêmes à combler les interstices, à «ponctuer» le texte. Bref, le poète pratique une écriture concise qu'on nommera plus tard le minimalisme poétique.

Autour de Françoise Sagan indélébile constitue, sans aucun doute, le livre le plus surréaliste de Des Roches. S'y trouvent regroupés plusieurs textes de facture fort différente, mais tous traversés par une sexualité leste. Le livre s'ouvre sur quelques poèmes isolés suivis de: «A Françoise Sagan indélébile»6, sous forme poétique; «Une prochaine roue», texte en prose; «les Contes d'haut», poèmes très formalistes; «Une page du livre des villes / Hommage à Paul Klee», un seul poème; «Brian Mobile», une suite de cinq poèmes entièrement rédigée en anglais; «la Princesse radiophonique (inachevé)», texte en prose; «l'Architexte

6 François Charron écrira dans la «Postface» du recueil: À l'intérieur du volume, "À Françoise Sagan indélébile» est le «morceau» qui m'apparaît le plus significatif. Je le considère comme l'un des textes surréalistes les plus importants de notre génération (p. 92). De même, Pierre Nepveu, dans le numéro 18 de Lettres québécoises (printemps 1980), reconnaît la grande valeur de ce texte. 
dur», poésie exploratoire 7 et, enfin, «Space-opera (deuxième temps: prose)», récit de science-fiction entremêlé de propos sur l'anticipation.

Imaginons un amas plutôt hétéroclite de textes dont la principale préoccupation demeure l'exploitation maximale de certains jeux et nous aurons une bonne idée du recueil. Par exemple, Des Roches multiplie les mots-valises, lorsqu'il ne coupe tout simplement pas un mot en deux. De même, il s'amuse à décloisonner le texte poétique en lui appliquant une mise en page habituellement réservée au domaine scientifique. Ajoutons à cela une recherche sonore très poussée (l'ombre de Claude Gauvreau plane sur ces textes), des images provocatrices et ironiques, de la pseudo science-fiction, quelques pointes anti-cléricales et, ce qui apporte une certaine unité à ce fouillis exploratoire, l'omniprésence d'une isotopie sexuelle:

je plonge dans mon vagin histoire d'ouvrir la bouche un peu Françoise Sagan en bouteilles non retournables qui vous font épargner l'argent que vous mettrez sur vos condoms de chez Dior au réveil au réveil je pense à elle écartelée sur la galaxie comme un merveilleux museau de rat (p. 173)

La nouvelle génération poétique des années soixante-dix nous a habitués aux références culturelles et intertextuelles de toutes sortes, et Des Roches ne fait pas exception à la règle. Se côtoieront donc les Rolling Stones, Sherlock Holmes, Néfertiti, Camus, Mozart, Jeanne d'Arc et une multitude d'autres noms propres renvoyant à autant d'époques et de réalités différentes. Somme toute, Autour de Françoise Sagan indélébile est un recueil de textes fort disparates, misant tantôt sur le signifiant, tantôt sur le signifié. Si le projet est indubitablement audacieux, le résultat est moins convaincant. Quoi qu'il en soit, il s'agit sûrement du livre le plus fou de Des Roches.

\section{Le corps certes et encore!}

Des Roches met souvent en scène un personnage écrivain qui relate ses prouesses sexuelles dans des descriptions précises, utilisant un langage direct. Dans le Corps certain, on apprend que les corps sont violemmeñt masturbés matins et soirs, et un personnage dira: Je t'ai plantée des heures et des. Au dire de ce narrateur, certaines de ces pages ressemblent aux acrobaties excessives qu'on retrouve dans les livres de Sade. Heureusement, Des Roches ne se prend pas au sérieux. Dans une note en bas de page, il fait intervenir $\mathrm{M}-$, le personnage principal de Reliefs de l'Arsenal, qui, après avoir constaté la fréquence et la durée des actes sexuels dans le Corps certain, soulève la question suivante: ou est la vérité physiologique? (fit $M-$ ): on peut tout dans la poésie, et fausser le corps n'est pas la moindre des choses. Avec un clin d'œil au mouvement féministe, Des Roches fait dire à un des personnages de Reliefs de l'Arsenal: «Mais voyons», sourit-il en se regardant, non sans un certain plaisir, bandé, dans

$7 \quad$ Auquel ne sont pas sans s'apparenter certains passages d'écRiturEs (1980) de Paul-Marie Lapointe. 
la glace, «ne tombons pas dans la pornographie...» (p. 35). Et c'est à notre tour de sourire.

Le regard, le voyeurisme et la masturbation sont des thèmes récurrents chez Des Roches, et les fantasmes sont régulièrement réfléchis par de multiples miroirs. Ainsi, un couple qui fait l'amour en se regardant dans un miroir donne l'impression qu'il y a quatre personnes en train de forniquer. Dans Reliefs de l'Arsenal, un officier en civil, buvant une boisson inconnue, explique le nouveau procédé de copulation mis au point par ses supérieurs: nous n'avons besoin que de miroirs. Une merveilleuse méthode de reproduction par un complexe jeu de miroirs sur des masses vivantes (jusqu'à ce stade informes). Nous nous amusons un peu évidemment (p. 29-30), et démontre ses objets de travail. Le narrateur du Soleil tourne autour de la terre, lui, aime s'imaginer s'imaginant, ce qui complique d'autant son rapport à la sexualité. Il poursuit en mentionnant qu'il pratique une sexualité à caractère infantile, mais il est conscient de ses diverses perversions: Il s'agit maintenant qu'elle accepte qu'on lui glisse un doigt dans l'anus, un doigt dans le vagin, qu'on masse sesfesses et qu'on lèche avec toute l'énergie du désespoir. Je suis un jouet essentiellement corrompu (p. 45).

Les récits de Des Roches présentent toujours des couples en difficulté, qui ne se rejoignent que dans la baise sauvage, dénuée de tendresse. Le narrateur-type est un écrivain célibataire qui refuse les liaisons durables et passionnées; son désir premier est de faire l'amour avec le plus grand nombre de filles possible, car chacune a ses particularités et dégage un parfum singulier. Les ruptures deviennent donc monnaie courante, et le narrateur profite de ces scènes pour nourrir ses fictions. Dans la Promenade du spécialiste, un personnage féminin lance, offusqué: Mais tu écris pendant que je souffre! Eh oui, c'est un fait. Peut-on alors affirmer que la poésie de Des Roches évoque, avec romantisme, le malaise d'aimer? Les Lèvres de n'importe qui et l'Observatoire romanesque ne revendiquent-ils pas le statut de lettres d'amour! Peut-être, mais nous sommes très éloignés du sentimentalisme fleur bleue des romans Harlequin, car derrière la complexité de la vie de couple, se faufilent deux obsessions manifestes, le sexe et le texte.

Des Roches se plaît à comparer la jouissance sexuelle à la jouissance textuelle, car, comme chez plusieurs auteurs des Herbes rouges, l'acte d'écrire est d'abord physique et, à ce titre, il jouit pleinement de tous les attributs du corps. Les deux sont intimement liés au point qu'il n'y aurait plus de différences notables entre ces deux plaisirs. Dans l'extrait suivant, le texte apparaît comme le résultat de savantes caresses qui relèvent d'un processus masturbatoire éloquent:

le texte se déroule en longues bandes grises entre leurs fesses car ils désirent et obtiennent confus le produit le texte procédé par petites touches hésitantes, particulièrement lentes (des jours que jouir est douloureux ou impossible et écrire ne fait que consommer que plus de 


\section{cigarettes) (le Corps certain)}

L'acte de lecture est lui aussi corporel et renforce l'équation sexe/texte: voulezvous lire le corps certain en remuant les lèvres? [en note: je ne puis m'empêcher de dire qu'en outre le pénis remue les lèvres en passant (n'est-il pas là pour qu'on lui parle?) car il est là un peu pour qu'on le parle] (le Corps certain). Le corps devient alors lui-même une fiction textuelle. On retrouve dans l'Observatoire romanesque un vers humoristique qui s'applique autant à l'acte d'écrire qu'à l'acte d'amour. J'ai beaucoup parlé et je parle toujours (ben d' la mine dans l' crayon!) (p. 5).

Le narrateur semble avoir une fixation sur les seins qui apparaissent systématiquement dans tous les recueils, à l'exception peut-être du recueil Pourvu que ça ait mon nom, dans lequel on retrouve la confession suivante:

Mon attention s'est récemment déplacée des seins aux fesses "comme vers un lieu de célébrations plus glorieux», ce qui n'est pas tout à fait juste, mais néanmoins témoigne d'une certaine recherche du "copieux». Cultiver les fesses, (p. 43).

Il suffit de lire les deux derniers textes de Des Roches pour se rendre compte de l'aspect éphémère de cette transition; nous n'avons jamais lu autant de métaphores et de comparaisons sur les seins, même dans les textes surréalistes. Il faut avouer que, parfois, les résultats sont fort heureux. Un seul exemple suffit à nous convaincre: La première chose qu'elle dit en s'éveillant fut: "Mes seins n'ont plus le même poids.» Je m'en assurai. Ses seins étaient en effet plus légers que l'air et s'avalaient très facilement (le Soleil tourne autour de la terre, p. 31$)^{8}$.

\section{Narration dites-vous, navré mais)}

Des Roches n'a pas hésité à employer certains procédés narratifs (personnages, dialogue, narrateur, quête, etc.) dans ses recueils de poèmes et il a même publié des récits qui, on s'en doute, n'ont rien de classique. Dans Reliefs de l'Arsenal ainsi que dans le cycle Space-opera, il présente un texte respectant les principales contraintes de la science-fiction (on y retrouve des robots, des astronefs, des êtres difformes, etc.), mais dont il est presque impossible de résumer l'histoire particulièrement brouillée. On ne sait pas trop où l'on va et les personnages se transforment sous nos yeux; de plus, le récit est constamment entrecoupé de propos théoriques sur l'anticipation. On conçoit bien que le but de l'auteur n'est pas de raconter une histoire, mais de déjouer les codes narratifs

8 Aparté ironique: Des Roches demeurant fidèle, dans tous ses écrits, au «thème» de la sensualité, nous devons avouer que relire, en quelques jours, son œuvre entière (en vue de la circonstance dite à la note 1) n'a pas été sans affecter notre propre libido. Si bien qu'à l'instar du narrateur de la Publicité discrète, nous nous délectons à trouver humide la plus grossière métaphore. 
usuels. Le lecteur, désorienté, ne sait plus sur quel pied danser; ironiquement, un personnage de «Space-opera (deuxième temps: prose)», venant rendre compte de la situation à son chef, dira: Le plan fonctionne à la perfection, chef, c'est la confusion la plus complète dans notre camp ou celui de l'ennemi, je ne saurais dire (p. 225). Des Roches apprécie beaucoup ce genre de clins d'œil! Le lecteur, quant à lui, doit faire confiance à l'auteur et ne pas chercher d'emblée à tout comprendre. Il espère cependant que les morceaux du puzzle se mettront finalement en place, et qu'à une deuxième lecture, il savourera davantage les méandres du texte. Pour lire Des Roches, il faut savoir apprécier son humour et reconnaître sa virtuosité à pervertir un code. Ses récits n'ont rien de linéaire ou de conventionnel, mais on en retire un plaisir certain, ne serait-ce que par les commentaires des personnages, les situations saugrenues ou les chocs résultant des brisures de rythme dans la narration.

Son personnage $\mathrm{M}$ - est un être androgyne (ou à tout le moins un travesti) qui ramène constamment les discussions à des considérations théoriques ou sexuelles, et certains de ses énoncés s'appliquent littéralement à l'écriture de Des Roches: Mon texte a l'embarrassante habitude de se replier à tout moment, se décalquer, se dédoubler (même plus, vous le remarquerez, de concordance des temps!) (Reliefs de l'Arsenal, p. 56). Nous le répétons, l'intérêt de ces récits ne réside pas dans l'histoire, mais dans la construction, la façon de la raconter; ainsi, les différents réseaux se répondent et se confondent pour le plus grand plaisir de l'auteur et, bien sûr, du lecteur. Sandra, un des personnages féminins du texte «Space-opera (deuxième temps: prose)» (ou est-ce tout simplement le même personnage qui change de nom?), explique à Henri le trait le plus intéressant de l'écriture qu'ils ont découverte, laquelle témoigne sûrement d'une civilisation supérieure:

- En effet, de même que chez les Égyptiens antiques, où l'hiéroglyphe prenait un autre sens selon le déterminatif précis qu'on plaçait devant, ceux-ci, hautement plus sophistiqués, et sans aucun moyen mécanique ou linguistique apparent, ceux-ci prennent autant de sens différents qu'il y a de personnes différentes qui les lisent. Ainsi cette plaque peut être un texte de loi ou un poème, selon que le lecteur est un magistrat ou un sot.) (p. 74-75)

Comme Des Roches n'hésite pas à s'immiscer dans ses fictions, en donnant son nom à un personnage, il en résulte un problème de lecture: doit-on lire ses textes comme des mémoires, des confidences ou des fictions burlesques? Des Roches serait-il le Superman du sexe comme il le laisse entrevoir dans ses livres? Dans son dernier recueil, par exemple, la rose des vents constituée par les initiales des filles qu'il a connues, au sens biblique du terme, contient-elle de vrais noms ou Des Roches s'amuse-t-il encore aux dépens du pauvre lecteur incrédule et naïf? Ce genre de tergiversation ne nous intéresse guère, puisque le texte se justifie par lui-même, et que les auteurs n'ont à intervenir que pour éclairer les conditions de production de leur époque. Nous ne voulons pas basculer dans l'analyse d'auteur — heureusement pour lui —, analyse qui, on l'a 
constaté à maintes reprises, reflète davantage les problèmes particuliers de l'analyste - heureusement pour nous.

Entre ses premières publications et le Soleil tourne autour de la terre, l'univers poétique de Des Roches s'est considérablement transformé. Bien sûr, on retrouve dans son dernier livre quelques références surréalistes telles que la douleur des autres / Capitales de la couleur (p. 13). Mais il n'y a, nous semblet-il, aucune trace d'écriture automatique, de hasard objectif ou d'objectivité narrative; si influence il y a, elle se situe au niveau de la psychanalyse et de la sexualité apprêtée à la sauce théorique moderne. Quant aux images, elles vacillent entre l'effet inattendu (Nous possédons huit doigts à chaque main, p. 11), et la plus grande simplicité (chacun de mes souvenirs [...] se découpe comme un camembert, p. 26). Les jeux sur le signifiant et le signifié, bien qu'en moins grand nombre, surgissent encore, ici et là, et malheureusement, ils n'ont pas tous le charme du vers suivant: Si le soleil coulait dans son cou, c'est que sa blouse blousait (p. 25).

Au niveau formel, ce sont d'abord les répétitions lexicales et syntaxiques qui nous frappent. Procédés constants dans tout le recueil, mais qui ont le très grand mérite de ne jamais agacer. Le ton et l'effet produit décuplent la force des énoncés. C'est peut-être aussi une façon de peindre la routine quotidienne qui s'installe dans la vie de couple. Le Soleil tourne autour de la terre raconte l'histoire avortée d'un couple et la culpabilité qui rejaillit sur le narrateur. Celuici s'accuse d'abord d'avoir peur, puis d'avoir faim et, plus loin, il spécifiera avoir peur du vide (p. 52). Il semble évident que le narrateur a d'abord peur de luimême, de son incapacité à construire des liaisons durables, de son manque de sensibilité. Et, un peu à la manière d'un enfant, il se retrouve dans la position paradoxale de celui qui craint d'être puni et qui jouit de cette même crainte: $S i$ c'est le désir tout seul, demandai-je, le désir tout nu plutôt que l'amour, mon amour, comment me puniras-tu? (p. 39). Le narrateur avoue d'ailleurs vers la fin du recueil: J'aime, j'aime jamais (p. 71). Ici, le jeu sur le signifiant a de terribles répercussions sur le signifié. Un autre procédé que l'auteur affectionne est la juxtaposition d'un même verbe employé à deux temps différents. Comme si le narrateur ne pouvait trancher entre le présent et le passé, il se justifie de la façon suivante: Je préfère l'imparfait au présent de l'indicatif, le fait accompli et les événements faciles, dans la mesure du possible (p. 14). On peut en déduire que les tensions et événements passés ont encore des répercussions aujourd'hui. Ce procédé revient à sept reprises. Rappelons que, dans les Lèvres de n'importe qui, un personnage affirmait: il se répète et ça fait le style et l'accessoire et le souffle (p. 9).

Dans le Soleil tourne autour de la terre, le Soleil prend le visage inquiétant du drame, de l'événement difficile. Il devient, en quelque sorte, l'étoile annonçant la mauvaise nouvelle, contrairement à l'étoile des bergers. Le narrateur poursuit le renversement des valeurs axiologiques usuelles par le biais de l'apposition métaphorique suivante: la détresse (le soleil de ma vie!) (p. 71). Le syntagme, très banal, tu es le soleil de ma vie acquiert ici une dimension 
nouvelle. Le narrateur croit qu'il est en quête de la femme idéale, mais ne poursuit-il pas plutôt la répétition de la formule qu'il connaît, formule qui dit la suite infinie de ses liaisons impossibles, qui le conduit inévitablement au désarroi et, peut-être même, à la jouissance. Freud l'a bien démontré, notre première aventure amoureuse (avec notre mère) aboutit à l'échec: La mer était au bout de la route et, dans la mer, il y avait un hôtel habité par l'illusion parfaite (p. 40). Pour avoir trompé si longtemps l'humanité, le Soleil tournant autour de la terre ne figure-t-il pas, lui aussi, l'illusion parfaite?

Roger Des Roches est, avec Yolande Villemaire, l'écrivain de sa génération qui fait le plus ouvertement référence au surréalisme. Il poursuit certaines visées esthétiques de ce courant avant-gardiste français des années vingt et trente, même s'il apparaît beaucoup plus influencé par les surréalistes québécois de la fin des années quarante, notamment Paul-Marie Lapointe et Claude Gauvreau. À cause de circonstances historiques et sociales largement commentées depuis, le surréalisme a franchi les portes du Québec avec quelques décennies de retard, mais 1948 allait marquer notre histoire littéraire, car, pour la première fois, un groupe de producteurs s'affranchissait des autorités religieuse et politique pour réclamer sa liberté d'expression.

Peu importe la voie qu'il suit, Des Roches semble s'écarter des lieux institutionnels de légitimation. Ses recherches le poussent constamment vers la nouveauté, à contre-courant des modes d'écriture, même si parfois il les engendre. Ses exigences et son travail formel rebutent le lecteur moyen ainsi que la critique en général. Par son style d'écriture, Des Roches se place volontairement en marge du milieu littéraire québécois et s'avère lui-même peut-être la cause du silence qui l'a si longtemps entouré. 